\title{
SAR Image Autofocus By Sharpness Optimization: A Theoretical Study
}

\author{
Robert L. Morrison, Jr., Member, IEEE, Minh N. Do. Member, IEEE, and David C. Munson, Jr., Fellow, IEEE,
}

\begin{abstract}
Synthetic aperture radar (SAR) autofocus techniques that optimize sharpness metrics can produce excellent restorations in comparison with conventional autofocus approaches. To help formalize the understanding of metric-based SAR autofocus methods, and to gain more insight into their performance, we present a theoretical analysis of these techniques using simple image models. Specifically, we consider the intensitysquared metric, and a dominant point-targets image model, and derive expressions for the resulting objective function. We examine the conditions under which the perfectly focused image models correspond to stationary points of the objective function. A key contribution is that we demonstrate formally, for the specific case of intensity-squared minimization autofocus, the mechanism by which metric-based methods utilize the multichannel defocusing model of SAR autofocus to enforce the stationary point property for multiple image columns. Furthermore, our analysis shows that the objective function has a special separble property through which it can be well approximated locally by a sum of 1-D functions of each phase error component. This allows fast performance through solving a sequence of 1-D optimization problems for each phase component simultaneously. Simulation results using the proposed models and actual SAR imagery confirm that the analysis extends well to realistic situations.
\end{abstract}

Index Terms - synthetic aperture radar, autofocus, sharpness optimization, sparsity condition, iterative methods.

\section{INTRODUCTION}

I $\mathrm{N}$ synthetic aperture radar (SAR) imaging, inaccurate range measurements or signal propagation through media with unknown spatially-varying propagation velocity can cause demodulation timing errors in the radar receiver. The result of the demodulation errors is undesired Fourier phase shifts in the imaging data that cause the formed imagery to be improperly focused. Specifically, the effect of the errors frequently may be modeled by a one-dimensional phase error function added to the Fourier phase of each column of the imaging data [1]. SAR autofocus encompasses the class of image restoration techniques for correcting the unknown phase aberrations using the defocused data and assumptions about the underlying scene.

Many solutions to the SAR autofocus problem have been proposed [1]-[14]. A widely-utilized autofocus technique is Phase Gradient Autofocus (PGA), which employs the basic

R. L. Morrison, Jr. is with the Department of Electrical and Computer Engineering and the Coordinated Science Laboratory, University of Illinois at Urbana-Champaign, IL 61801 USA (e-mail: rlmorris@ifp.uiuc.edu).

M. N. Do is with the Department of Electrical and Computer Engineering, the Coordinated Science Laboratory, and the Beckman Institute, University of Illinois at Urbana-Champaign, IL 61801 USA (e-mail: minhdo@uiuc.edu).

D. C. Munson, Jr. is with the Department of Electrical Engineering and Computer Science, University of Michigan, Ann Arbor, MI 48109 USA (email: munson@umich.edu).

This work was supported by the National Science Foundation under Grant CCR 0430877. principles of inverse filtering, and augments them with an innovative iterative windowing and averaging process to estimate the phase error [1], [5]-[8]. PGA typically produces an accurate approximation of the phase error for a variety of errors. Exceptional restoration quality has been observed through the use of a class of autofocus methods that optimize image sharpness metrics [9]-[15]. In these metric-based autofocus methods, the compensating phase estimate is selected through an optimization algorithm to maximize a particular sharpness metric evaluated on the defocused image intensity. Examples of the optimization approaches used in these methods include gradient-descent techniques [9], coordinate direction searches [11], and monotonic iterative algorithms [10], [12], [16]. The use of metric-based autofocus algorithms sometimes produces superior restorations in comparsion with the conventional PGA method in experiments using synthetic and actual SAR imagery [15], [17].

Much of the current understanding of metric-based autofocus techniques is based on intuition and results from processing data sets. Thus, it is of interest to obtain a clearer understanding of the performance of these autofocus methods. Such an understanding might enable the powerful restoration ability of these methods to achieve more widespread use. Sharpness metrics were first explored in 1974 by Muller and Buffington for the real-time correction of phase distortions in telescopic imaging systems [18]. Some recent work was done by Fienup et al. in justifying the the use of particular metrics for SAR given prior assumptions on the underlying image model [9]. The goal of our work is to gain further insight into metric-based methods for SAR autofocus through studying a simple dominant point-targets image model. Such a model has been used to motivate existing autofocus approaches [1], [5], [9], [11]. Considering the intensity-squared metric, we derive expressions for the objective function as a function of the parameters of the proposed models and also the unknown phase errors. Our expressions, which describe the variation of the metric along the phase-error coordinate directions, are used to determine the conditions under which the perfectly focused SAR image models correspond to stationary points of the objective function (i.e., points of zero gradient) [19][21]; these are points where the optimization algorithms used in metric-based autofocus terminate.

Because the phase error is a one-dimensional function of the cross-range frequency coordinate, each range bin (i.e., column of the image) is defocused by the same blurring kernel; we denote this as the multichannel defocusing model of SAR autofocus [22], [23]. It has been observed that autofocus approaches generally require multiple columns of the defocused image to produce an accurate estimate of the 
phase error function; this is true of metric-based methods as well [9], [11]. Thus, it is not the sharpness metric or image model alone that allows the image to be properly restored, but also the redundancy of the defocusing operation on each image column. Our key contribution is that we conclusively demonstrate how the assumption of the multichannel defocusing model is exploited in metric-based SAR autofocus methods. Our analysis provides formal justification for the finding that one-dimensional point-target models, such as a single column of the perfectly focused image, generally do not correspond to stationary points of the objective function. We demonstrate the mechanism by which accurate estimation of the phase error is possible when multiple image columns are available for the specific case of intensity-squared minimization autofocus; the (objective function) minima from multiple columns reinforce each other, or average, to form a stationary point at the perfectly focused phase estimate. It is through this averaging mechanism that metric-based methods implicitly use the multichannel assumption and correctly estimate the phase error.

We also demonstrate that the objective function has a special structure through which it can be well approximated locally by a sum of 1-D functions of each phase error component. Thus, we show that, within a small neighborhood, the multivariate objective function is a separable function of the phase perturbations. In particular, we demonstrate that for sparse images the separable approximation is highly accurate within a radius of the perfectly focused solution; this radius can be expressed as a function of the number of dominant point targets and the number of resolution cells. The separable property allows fast optimization using a simultaneous coordinate descent approach, where a sequence of 1-D optimization problems is solved for each phase component simultaneously, and underlies the success of efficient algorithms for metricbased SAR autofocus [10], [12].

The organization of this paper is as follows. Section 2 presents a statement of the SAR autofocus problem. The image model and sharpness metric used in our analysis are defined, and we state the optimization problem for metric-based SAR autofocus methods. In Section 3, we derive expressions for the intensity-squared objective function using one-dimensional (single-column) image models. Section 4 extends the analysis to two-dimensional (multicolumn) images. Using a stochastic image model where the parameters of the model are selected according to a particular distribution, we demonstrate that as multiple image columns are combined to form the objective function, the gradient at the perfectly focused phase estimate approaches zero, satisfying the stationary point condition. In Section 5 , we show that the multivariate objective function is approximately a separable function of the phase perturbations locally, and discuss how this property enables efficient approaches for perfoming the optimization. In Section 6, we present numerical experiments using actual SAR imagery to validate the analytical results and show that the analysis extends well to realistic situations.

\section{Problem Setup}

\section{A. The Autofocus Problem}

Let $\boldsymbol{g} \in \mathbb{C}^{M \times N}$ be the perfectly focused SAR image. After range compression (inverse 1-D DFT in the range direction), the collected Fourier imaging data $G \in \mathbb{C}^{M \times N}$ are related to $\boldsymbol{g}$ through a 1-D DFT applied to each column [24]:

$$
g[m, n]=D F T_{k}^{-1}\{G[k, n]\} \stackrel{\text { def }}{=} \frac{1}{M} \sum_{k=0}^{M-1} G[k, n] e^{j 2 \pi k m / M} .
$$

Here, the row index $m(m=0,1, \ldots, M-1)$ corresponds to the cross-range dimension, the column index $n(n=0,1, \ldots, N-$ 1) to the range dimension, and $D F T_{k}^{-1}$ denotes an inverse DFT with respect to the cross-range frequency index $k$ ( $k=$ $0,1, \ldots, M-1)$.

In practice, $\boldsymbol{G}$ is corrupted by multiplicative phase errors that produce a defocused image. A mathematical model relating the defocused Fourier imaging data $\tilde{G}$ to the focused data $G$ is [1]:

$$
\tilde{G}[k, n]=G[k, n] e^{j \phi_{e}[k]},
$$

where $\phi_{e} \in[-\pi, \pi)^{M}$ is a 1-D Fourier phase error function. Using (1) and (2), the defocused image is related to the perfectly focused image through

$$
\tilde{g}[m, n]=D F T_{k}^{-1}\left\{D F T_{m^{\prime}}\left\{g\left[m^{\prime}, n\right]\right\} e^{j \phi_{e}[k]}\right\} .
$$

Autofocus algorithms form an estimate $\hat{\phi}_{e}$ of the phase error function to correct the defocused imaging data, forming the restored image

$$
\hat{g}[m, n]=D F T_{k}^{-1}\left\{\tilde{G}[k, m] e^{-j \hat{\phi}_{e}[k]}\right\} .
$$

\section{B. SAR Image Models}

We utilize a dominant point-targets model for the SAR image. Such a model can be considered as a rough representation of SAR and ISAR images when there is strong return from isolated scatterers. This simple, yet analytically tractable model has been used to motivate existing autofocus approaches [1], [5], [9], [11]. We consider the sparse discrete signal $\boldsymbol{g}_{s} \in \mathbb{C}^{M \times N}$, where each column of $\boldsymbol{g}_{s}$, representing a fixed range coordinate $n$ or a single range bin, contains $P$ weighted impulses:

$$
g_{s}[m, n]=\sum_{p=0}^{P-1} a_{p}[n] e^{j \theta_{p}[n]} \delta\left[m-m_{p}[n]\right],
$$

where $\delta[m]$ is the discrete unit impulse signal. Each impulse represents a point target with magnitude $a_{p}[n]$, spatial-domain phase shift $\theta_{p}[n]$, and location $m=m_{p}[n]$. We assume that the number of dominant targets is much smaller than the number of resolution cells (pixels) per range bin: $P \ll M$. 


\section{Image Sharpness Metrics}

Metric-based autofocus algorithms use image sharpness metrics to evaluate the degree of focus. Because of the pointlike nature of the SAR image model, maximizing sharpness is found to increase the image focus. The aim of these methods is to determine the image in the search space (4) with maximum sharpness, as measured by a particular metric.

The metrics we consider are additive in the sense that the value of the metric, or cost, is a sum of contributions from each pixel individually. We define $\varphi: \mathbb{R}^{+} \rightarrow \mathbb{R}$ as a concave cost function operating on the intensity of each pixel $I[m, n] \stackrel{\text { def }}{=}$ $|g[m, n]|^{2}$. In this paper, we study the intensity-squared cost function [9], [11], [12], [14], [18]

$$
\varphi(I)=-I^{2} .
$$

The metric $\mathcal{C}: \mathbb{C}^{M \times N} \rightarrow \mathbb{R}$ maps the image $\boldsymbol{g}$ to a sharpness cost:

$$
\mathcal{C}(\boldsymbol{g})=\sum_{n=0}^{N-1} \sum_{m=0}^{M-1} \varphi\left(|g[m, n]|^{2}\right)
$$

Due to the concavity of $\varphi$, sharpening the image (increasing the variance of the pixel intensities about their mean) decreases the value of the $\operatorname{cost} \mathcal{C}$ [9]. Therefore, we wish to minimize the metric $\mathcal{C}$ (maximize sharpness).

\section{SAR Autofocus as an Optimization Problem}

The objective function for the defocused image $\tilde{\boldsymbol{g}}, f_{\tilde{g}}$ : $[-\pi, \pi)^{M} \rightarrow \mathbb{R}$, is defined as

$$
f_{\tilde{g}}(\phi)=\mathcal{C}\left(\tilde{\boldsymbol{g}}_{\phi}\right),
$$

where $\tilde{g}_{\phi}[m, n]=D F T_{k}^{-1}\left\{\tilde{G}[k, m] e^{-j \phi[k]}\right\}$ is an image in the search space (4). In other words, $f_{\tilde{g}}(\phi)$ is the metric evaluated in the space of images formed from $\tilde{\boldsymbol{g}}$ by applying a particular Fourier phase correction function $\phi$.

Metric-based autofocus methods employ optimization algorithms, which act on $\phi$ to determine a minimizer of $f_{\tilde{g}}$. However, the optimization techniques used in these methods may determine local minimizers of $f_{\tilde{g}}$ [19], [25]. Therefore, we are interested in the behavior of the objective function locally about the perfectly focused image. We introduce the function

$$
f_{g}(\boldsymbol{\phi})=\mathcal{C}\left(\boldsymbol{g}_{\phi}\right)
$$

where $g_{\phi}[m, n]=D F T_{k}^{-1}\left\{G[k, m] e^{-j \phi[k]}\right\}$. The function $f_{g}$ is the objective function where the origin $\phi=0$ is defined with respect to $\boldsymbol{g}$ instead of $\tilde{\boldsymbol{g}}: f_{g}(\boldsymbol{\phi})=f_{\tilde{g}}\left(-\boldsymbol{\phi}_{\boldsymbol{e}}+\boldsymbol{\phi}\right)$.

The key in our analysis is to derive expressions for $f_{g}\left(\phi \boldsymbol{e}_{k}\right)$, where $\boldsymbol{e}_{k}$ is the $k$-th element of the standard basis for $\mathbb{R}^{M}$, i.e., $\boldsymbol{e}_{k}[m]=1$ if $m=k$ and 0 otherwise, and $\phi \boldsymbol{e}_{k}(\phi \in$ $[-\pi, \pi))$ is the $k$-th component of $\phi$, using the model $\boldsymbol{g}_{s}$ in (5); note that $\phi$ is a scalar and $\phi$ (in boldface) is a vector. Such expressions describe the objective function along the phaseerror coordinate directions $\left\{\boldsymbol{e}_{k}\right\}_{k=0}^{M-1}$. The expressions are used to determine the conditions under which $\boldsymbol{g}_{s}$ corresponds to a stationary point of the objective function. Stationary points are places where the gradient-based optimization algorithms in metric-based SAR autofocus terminate; such points satisfy the first-order necessary condition for optimality [19], [21].
The stationary point condition requires zero gradient at the origin of the objective function [19]:

$$
\nabla f_{g}(\mathbf{0})=\mathbf{0}
$$

where

$$
\left.\nabla f_{g}(\boldsymbol{\phi})\right|_{\boldsymbol{\phi}=\mathbf{0}}=\left[\left.\frac{\partial f_{g}\left(\phi \boldsymbol{e}_{0}\right)}{\partial \phi}\right|_{\phi=0}, \ldots,\left.\frac{\partial f_{g}\left(\phi \boldsymbol{e}_{M-1}\right)}{\partial \phi}\right|_{\phi=0}\right] .
$$

\section{AnAlysis of Single-COLUmN IMAGE Models}

\section{A. Approximate Expressions for the Objective Function}

In this section, we analyze one column of the dominant point-targets model $\boldsymbol{g}_{s}$ in (5), which represents a fixed range coordinate $n$ (i.e., a single range bin):

$$
g[m]=\sum_{p=1}^{P} a_{p} e^{j \theta_{p}} \delta\left[m-m_{p}\right] .
$$

We first characterize the effect on the image of perturbing a single component $\phi \boldsymbol{e}_{k}$ of the Fourier phase of $\boldsymbol{g}$. Such a characterization is then used to derive an approximate expression for the squared image intensity as a function of $\phi \boldsymbol{e}_{k}$, which leads directly to expressions for $f_{g}\left(\phi \boldsymbol{e}_{k}\right)$.

The perturbed image $\boldsymbol{g}_{\phi \boldsymbol{e}_{k}}$ is defined as the image formed by perturbing the $k$-th component of the Fourier phase of $\boldsymbol{g}$ by an amount $\phi$ (i.e., $\angle G[k]+\phi$ ):

$$
g_{\phi \boldsymbol{e}_{k}}[m] \stackrel{\text { def }}{=} D F T_{k^{\prime}}^{-1}\left\{D F T_{m^{\prime}}\left\{g\left[m^{\prime}\right]\right\} e^{j \phi \delta\left[k^{\prime}-k\right]}\right\} .
$$

This may be alternatively expressed as

$$
g_{\phi \boldsymbol{e}_{k}}[m]=g[m]+\varepsilon_{\phi \boldsymbol{e}_{k}}[m],
$$

where

$$
\varepsilon_{\phi \boldsymbol{e}_{k}}[m]=\left(e^{j \phi}-1\right) s_{k}[m]
$$

is the update to pixel $m$ due to $\phi$, and $s_{k}[m]$ is the subband image:

$$
s_{k}[m]=\frac{1}{M} G[k] e^{j 2 \pi k m / M} .
$$

The $\left(e^{j \phi}-1\right)$ term comes from subtracting out the $k$-th term in the Fourier sum where the phase has not been perturbed, and adding in a new term where the phase has been perturbed by $\phi e_{k}$.

We derive an approximate expression for the squared intensity of the perturbed image: $I_{\phi \boldsymbol{e}_{k}}^{2}[m]=\left|g_{\phi \boldsymbol{e}_{k}}[m]\right|^{4}$. Using (14),

$$
\begin{aligned}
I_{\phi \boldsymbol{e}_{k}}^{2}[m] & =\left|g[m]+\varepsilon_{\phi \boldsymbol{e}_{k}}[m]\right|^{4} \\
& =\left(\left(g[m]+\varepsilon_{\phi \boldsymbol{e}_{k}}[m]\right)\left(g^{*}[m]+\varepsilon_{\phi \boldsymbol{e}_{k}}^{*}[m]\right)\right)^{2} \\
& =|g[m]|^{4}+4 \Re\left\{|g[m]|^{2} g^{*}[m] \varepsilon_{\phi \boldsymbol{e}_{k}}[m]\right\} \\
& +2|g[m]|^{2}\left|\varepsilon_{\phi \boldsymbol{e}_{k}}[m]\right|^{2}+4 \Re\left\{\left|\varepsilon_{\phi \boldsymbol{e}_{k}}[m]\right|^{2} g^{*}[m] \varepsilon_{\phi \boldsymbol{e}_{k}}[m]\right\} \\
& +4\left(\Re\left\{g^{*}[m] \varepsilon_{\phi \boldsymbol{e}_{k}}[m]\right\}\right)^{2}+\left|\varepsilon_{\phi \boldsymbol{e}_{k}}[m]\right|^{4},
\end{aligned}
$$

where $\Re$ denotes the real part of the argument. We approximate (17) by retaining the first two terms; this is equivalent to the first-order Taylor series expansion of (17) about $\varepsilon_{\phi \boldsymbol{e}_{k}}[\mathrm{~m}]=0$ :

$$
I_{\phi \boldsymbol{e}_{k}}^{2}[m] \approx I^{2}[m]+4 \Re\left\{I[m] g^{*}[m] \varepsilon_{\phi \boldsymbol{e}_{k}}[m]\right\},
$$


where $I[m]=|g[m]|^{2}$ and $I^{2}[m]=|g[m]|^{4}$. The benefit of using an approximation is that the expression is linear in the image update $\varepsilon_{\phi \boldsymbol{e}_{k}}[\mathrm{~m}]$, which will result in a simplified and intuitive expression for the objective function. To justify that (18) is an accurate approximation, we show that $\left|\varepsilon_{\phi \boldsymbol{e}_{k}}[m]\right| \ll$ $|g[m]|$ at pixels where a target is present (i.e., $m=m_{p}$ ). Using $\left|\left(e^{j \phi}-1\right)\right| \leq|\phi| \leq \pi$, and $|G[k]| \leq \sum_{m}|g[m]| \leq P\|\boldsymbol{g}\|_{\infty}$ on (15) and (16), we have the upper bound

$$
\left|\varepsilon_{\phi \boldsymbol{e}_{k}}[m]\right| \leq \frac{P}{M}|\phi|\|\boldsymbol{g}\|_{\infty}
$$

for all $m$ and $k$. Thus if

$$
\frac{P}{M}|\phi| \ll 1,
$$

then the approximation in (18) is accurate. Note that this is true for the sparse model (12) where $P \ll M$. As an example, let $P=24, M=1024$, and $|\phi|=\pi$. Then $\frac{P}{M}|\phi| \approx$ $0.074 \ll 1$. Since $\left|\varepsilon_{\phi \boldsymbol{e}_{k}}[m]\right|$ decreases with decreasing $\phi$, the approximation (18) becomes especially good for small phase perturbations (e.g., $|\phi| \leq \frac{\pi}{4}$ ). Thus, the expression for the objective function will be highly accurate locally about the perfectly focused solution.

Using (15) and (18), the intensity-squared objective function evaluated for a single phase perturbation $\phi \boldsymbol{e}_{k}$ is expressed as

$$
\begin{aligned}
f_{g}\left(\phi \boldsymbol{e}_{k}\right) & =-\sum_{m=0}^{M-1} I_{\phi \boldsymbol{e}_{k}}^{2}[m] \\
& \approx-\sum_{m=0}^{M-1} I^{2}[m]-\Re\left\{\left(e^{j \phi}-1\right) z_{k}\right\},
\end{aligned}
$$

where

$$
z_{k}=4 \sum_{m=0}^{M-1} I[m] g^{*}[m] s_{k}[m]
$$

Note that (21) can be rewritten as

$$
f_{g}\left(\phi \boldsymbol{e}_{k}\right) \approx c_{k}-\left|z_{k}\right| \cos \left(\phi+\angle z_{k}\right)
$$

where $c_{k}=-\sum_{m=0}^{M-1} I^{2}[m]+\Re\left\{z_{k}\right\}$ is a constant given the perfectly focused image.

The expression (23) reveals that the behavior of $f_{g}\left(\phi \boldsymbol{e}_{k}\right)$ for every $k$ is described by a cosine function with an amplitude and phase shift dependent on the complex number $z_{k}$, which is a function of the perfectly focused image model. We note that expressions similar to (23) have been derived independently in [10] and [12] using different approximations and assumptions.

Using (12), we define (22) explicitly in terms of the model parameters:

$$
z_{k}=\frac{4}{M} \sum_{p=0}^{P-1} \sum_{l=0}^{P-1} a_{l}^{3} a_{p} e^{j \psi_{k}[l, p]}
$$

where

$$
\psi_{k}[l, p]=\theta_{p}-\theta_{l}+\frac{2 \pi}{M} k\left(m_{l}-m_{p}\right) .
$$

The contribution of the spatial-domain phases and the locations of the pair of targets at $m=m_{l}, m_{p}$ resides exclusively within the parameter $\psi_{k}[l, p]$.
The expression (24) shows that $z_{k}$ is generally not realvalued, so $\angle z_{k} \neq 0$ in general. For the stationary point condition of $f_{g}\left(\phi \boldsymbol{e}_{k}\right)$ given in (23) to be satisfied, a necessary and sufficient condition is that $\angle z_{k}=0$ for all $k$. The presence of the $\angle z_{k}$ phase shift causes the minima of $f_{g}\left(\phi \boldsymbol{e}_{k}\right)$ to be displaced from the origin, so that the perfectly focused image does not correspond to a stationary point.

We note that the expressions in (21) and (22) bear some similarity to to the expression for the gradient of the objective function of generalized sharpness metrics derived by Fienup in [9]. It can be shown that the derivative of (21) at $\phi=0$ is equal to the Fienup gradient evaluated at $\phi=0$; i.e., the approximate expression here has the same functional value and gradient as the exact objective function at the origin. This explains the observed similarity in the two expressions.

\section{B. Validation of the Approximate Expression}

Figure 1(a) shows the magnitude of a three-target realization of the image model (i.e., $P=3$ ) with $M=128$. The plot in Figure 1(b) shows the behavior of the objective function along the coordinate direction $e_{18}$ (selected as a representative example) on the interval $[-\pi, \pi)$ for the model in Figure 1(a). The exact numerically evaluated metric is displayed as a solid curve, and the approximate expression in (23) is displayed as a dashed curve. The approximate expression is observed to be in excellent agreement with the exact expression, particularly for small $\phi$. Similar agreement is found using other directions $e_{k}$ and other realizations of the model $g$.

In this example, the objective function in Figure 1(b) does not have a minimum at the origin (since the minimum of $f_{g}\left(\phi \boldsymbol{e}_{18}\right)$ is not at $\left.\phi=0\right)$, and applying the optimization to the perfectly focused image would produce an erroneous restoration. In general, metric-based methods cannot restore a single column of the SAR image. However, we will show that the image model in (5) with multiple image columns can be properly focused through these techniques. In the next section, we demonstrate that the combination of the objective functions arising from each column individually causes the origin of the objective function for the multicolumn image to approach a stationary point.

\section{Analysis of Multicolumn Image Models}

\section{A. Asymptotic Analysis}

In the previous section, we determined expressions for the objective function considering only a single image column. Since the metrics we consider are additive, the objective function evaluated for a multicolumn image can be expressed as the sum of the objective functions evaluated for each image column individually:

$$
f_{g}\left(\phi \boldsymbol{e}_{k}\right)=\sum_{n=0}^{N-1} f_{g^{[n]}}\left(\phi \boldsymbol{e}_{k}\right)
$$

where $\boldsymbol{g}^{[n]}$ denotes the $n$-th column of $\boldsymbol{g}$. Our goal is to show that when a large number of columns of the point-targets model are incorporated, the origin of the objective function approaches a stationary point. To quantitatively demonstrate 


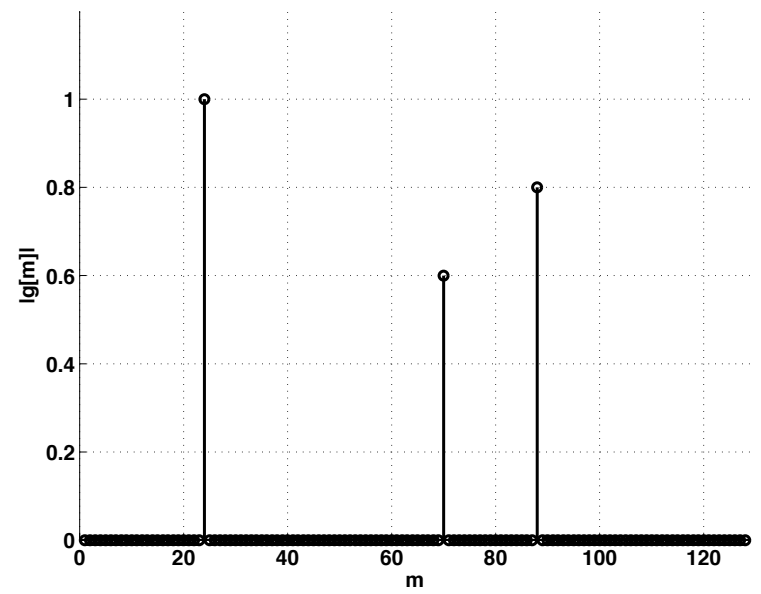

(a)

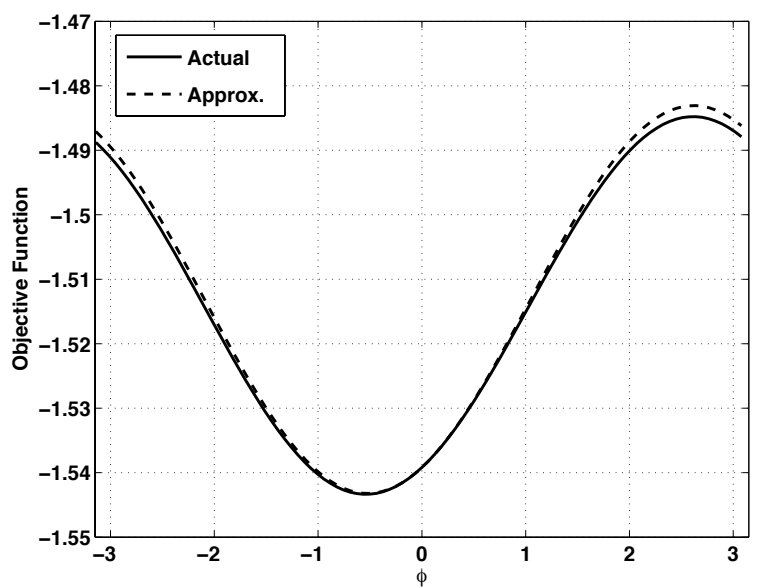

(b)

Fig. 1. An approximate expression for the objective function using the intensity-squared cost: (a) perfectly focused range bin model, and (b) plots of $\underset{f}{ }\left(\phi \boldsymbol{e}_{18}\right)$ versus $\phi \in[-\pi, \pi)$ for the model in (a), where the solid plot shows the exact numerically evaluated metric, and the dashed plot uses the approximate expression in (23).

this, we employ a stochastic image model for $\boldsymbol{g}$ by analogy with (5):

$$
g[m, n]=\sum_{p=0}^{P-1} A_{p}[n] e^{j \Theta_{p}[n]} \delta\left[m-m_{p}[n]\right],
$$

where $A_{p}[n]$ and $\Theta_{p}[n]$ are random variables characterizing the target magnitudes and spatial-domain phases, respectively. The following statistical assumptions are used in the analysis:

- The magnitudes $A_{p}[n]$ are independent and identically distributed (i.i.d.), with a distribution on $\mathbb{R}^{+}$having a finite variance.

- The spatial-domain phases $\Theta_{p}[n]$ are independent and uniformly distributed between $-\pi$ and $\pi$.

- The random variables $A_{p}[n]$ and $\Theta_{p}[n]$ are independent of each other.

The target locations $m_{p}[n]$ may be arbitrary, given that no two targets are assigned the same location: $m_{p}[n] \neq m_{q}[n]$ for all $p, q: p \neq q$. The random phase assumption is accurate for many scenarios where the surface roughness is on the scale of the radar wavelength [1], [26], [27]. In fact, the assumption has been shown to be important for SAR image reconstruction; similar to holographic imaging, random phase permits formation of high-resolution images from bandlimited, frequency-offset Fourier data [26].

Using the expression (21) in (26) yields

$$
\begin{aligned}
f_{g}\left(\phi \boldsymbol{e}_{k}\right) & \approx-\sum_{n=0}^{N-1} \sum_{m=0}^{M-1} I^{2}[m, n]-\Re\left\{\left(e^{j \phi}-1\right) \sum_{n=0}^{N-1} Z_{k}[n]\right\}, \\
& =C_{k}-\left|\sum_{n=0}^{N-1} Z_{k}[n]\right| \cos \left(\phi+\angle \sum_{n=0}^{N-1} Z_{k}[n]\right)
\end{aligned}
$$

where $Z_{k}[n]$ is the coefficient $z_{k}$ in (25) evaluated for range coordinate $n$ using the model (27):

$$
Z_{k}[n]=\frac{4}{M} \sum_{p=0}^{P-1} \sum_{l=0}^{P-1} A_{l}^{3}[n] A_{p}[n] e^{j \Psi_{k}[l, p, n]},
$$

$$
\Psi_{k}[l, p, n]=\Theta_{p}[n]-\Theta_{l}[n]+\frac{2 \pi}{M} k\left(m_{l}[n]-m_{p}[n]\right),
$$

and $C_{k}=-\sum_{m, n} I^{2}[m, n]+\Re\left\{\sum_{n} Z_{k}[n]\right\}$. Note that since $A_{p}[n]$ and $\Theta_{p}[n]$ are i.i.d. in $n, Z_{k}[n]$ is an i.i.d. sequence in $n$ for every $k$.

Define

$$
\Omega_{k}^{[N]}=\angle \sum_{n=0}^{N-1} Z_{k}[n]
$$

to be the phase shift associated with the $k$-th coordinate direction. The Strong Law of Large Numbers will be used to show that, as $N$ becomes large, the sum over $Z_{k}[n]$ converges to its expected value (scaled by $N$ ). We then will show that the expected value is real-valued, so that $\lim _{N \rightarrow \infty} \Omega_{k}^{[N]}=0$ for all $k$, demonstrating that $\phi=0$ is a stationary point of $f_{g}$. For fixed $k$, the Strong Law implies that with probability one [28]:

$$
\frac{1}{N} \sum_{n=0}^{N-1} Z_{k}[n] \rightarrow E_{A, \Theta}\left[Z_{k}\right] \text { as } N \rightarrow \infty,
$$

where $E_{A, \Theta}$ is the expected value with respect to $A$ and $\Theta$. Due to the uniform distribution on the spatial-domain phases, from (30) we see that $e^{j \Psi_{k}[l, p, n]}=1$ if $l=p$ and otherwise is uniformly distributed on the unit circle, so that

$$
E_{\Theta}\left[e^{j \Psi_{k}[l, p, n]}\right]= \begin{cases}1 & \text { for } l=p \\ 0 & \text { otherwise. }\end{cases}
$$

As a result, only terms in (29) where $l=p$ contribute to the expectation:

$$
\mu_{Z} \stackrel{\text { def }}{=} E_{A, \Theta}\left[Z_{k}\right]=\frac{4}{M} P E_{A}\left[A^{4}\right] .
$$

Therefore, for large $N$ we have

$$
\sum_{n=0}^{N-1} Z_{k}[n] \approx N \mu_{Z}
$$

which due to (34) is positive and real-valued. 
Using (35), the expression (28) for large $N$ becomes

$$
f_{g}\left(\phi \boldsymbol{e}_{k}\right) \approx C-N \mu_{Z} \cos (\phi),
$$

where $C=-\sum_{n, m} I^{2}[m, n]-N \mu_{Z}$. Thus, we see that the stationary point condition is satisfied (i.e., $\left.\frac{\partial f_{g}\left(\phi \boldsymbol{e}_{k}\right)}{\partial \phi}\right|_{\phi=0}=$ 0 for all $k$ ), and the metric is nondecreasing along each coordinate direction.

\section{B. Quantitative Analysis for a Finite Number of Columns}

Expressing the phase shift (31) in terms of the real and imaginary parts of $Z_{k}[n]$ :

$$
\Omega_{k}^{[N]}=\tan ^{-1}\left[\frac{\sum_{n=0}^{N-1} \Im\left\{Z_{k}[n]\right\}}{\sum_{n=0}^{N-1} \Re\left\{Z_{k}[n]\right\}}\right],
$$

where $\Im$ specifies the imaginary part of the argument. We approximate (37) using two observations. First, $\sum_{n=0}^{N-1} \Re\left\{Z_{k}[n]\right\} \gg \sum_{n=0}^{N-1} \Im\left\{Z_{k}[n]\right\}$, which is based on (35) being purely real; this implies

$$
\Omega_{k}^{[N]} \approx \frac{\sum_{n=0}^{N-1} \Im\left\{Z_{k}[n]\right\}}{\sum_{n=0}^{N-1} \Re\left\{Z_{k}[n]\right\}} .
$$

Furthermore, it can be shown that the expected value of the denominator in (38) is much greater than its standard deviation $^{1}$, so we make the approximation that only the variation in the numerator is significant, and approximate the denominator using its mean $\sum_{n=0}^{N-1} \Re\left\{Z_{k}[n]\right\} \approx N \mu_{Z}$ :

$$
\Omega_{k}^{[N]} \approx \sum_{n=0}^{N-1} \frac{\Im\left\{Z_{k}[n]\right\}}{N \mu_{Z}} .
$$

To determine how quickly the phase shifts tend to zero, we examine the variance as a function of $N$. Using (39), it can be shown that

$$
\sigma_{\Omega}^{2}[N] \stackrel{\text { def }}{=} \operatorname{Var}\left[\Omega_{k}^{[N]}\right] \approx\left(\frac{\lambda}{2} \frac{P-1}{P}\right) \frac{1}{N},
$$

where

$$
\lambda=\frac{E_{A}\left[A^{6}\right] E_{A}\left[A^{2}\right]-\left(E_{A}\left[A^{4}\right]\right)^{2}}{\left(E_{A}\left[A^{4}\right]\right)^{2}} .
$$

Since (39) is a sum of i.i.d. random variables with finite variance, by the central limit theorem $\Omega_{k}^{[N]}$ approaches a normal distribution with mean zero and variance $\sigma_{\Omega}^{2}[N]$ [29]. The key is noting that as the number of columns increases, the variance of the phase shifts decreases as $1 / N$.

We note that [30] uses a similar mathematical derivation to show, for the specific case of the shear averaging autofocus algorithm, that that the variance of the error in the phase estimate (under the shear averaging approach) decreases with the number of range bins as $1 / N$. It should be stressed that while the stastical assumptions and mathematical manipulations used in [30] and our work (i.e., the derivation leading up to (40)) are similar, the result in [30] is for a

${ }^{1} \operatorname{Var}\left[\Re\left\{Z_{k}[n]\right\}\right] \approx 16 / M^{2}\left(P E\left[A^{8}\right]+\left(P^{2} / 2-3 P / 2\right)\left(E\left[A^{4}\right]\right)^{2}+\right.$ $\left.P(P-1) E\left[A^{6}\right] E\left[A^{2}\right] / 2\right)$. The condition $E\left[\sum_{n} \Re\left\{Z_{k}[n]\right\}\right] \gg$ $\operatorname{StdDev}\left[\sum_{n} \Re\left\{Z_{k}[n]\right\}\right]=\sqrt{N \operatorname{Var}\left[\Re\left\{Z_{k}[n]\right\}\right]}$ holds when the target magnitudes are drawn from a Rayleigh distribution.

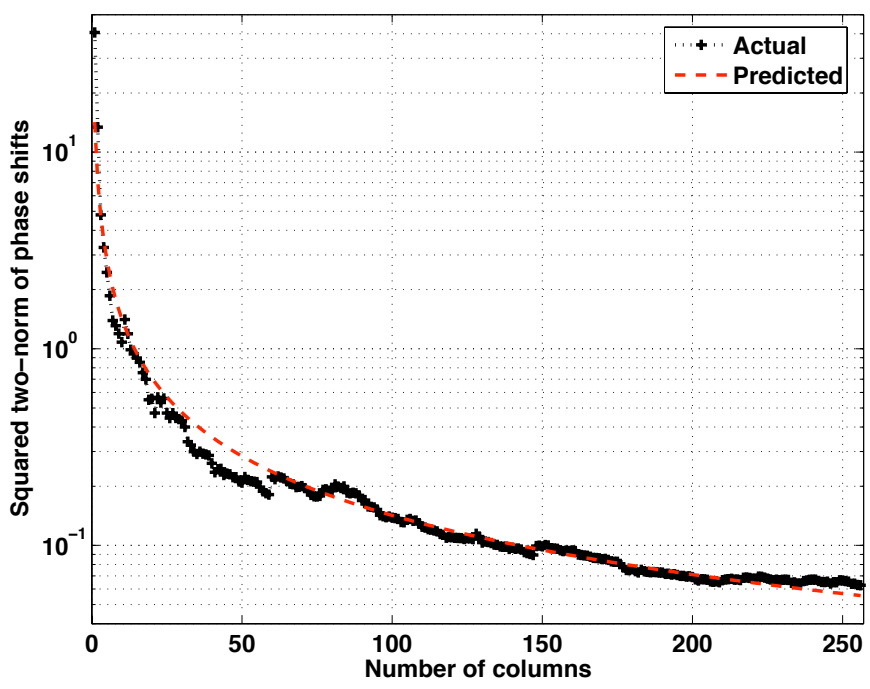

Fig. 3. Log-scale plot showing the squared $\ell_{2}$-norm of the phase shifts (i.e., deviations from $\phi=0$ ) as a function of the number of columns $N$ (plot with cross markers). The behavior as a function of $N$ is found to be proportional to $\frac{1}{N}$ (displayed in the dashed curve)

completely different autofocus algorithm that does not use sharpness metric optimization. The results in this paper are for the specific case of intensity-squared sharpness optimization, which to our knowledge have not been presented in the SAR autofocus literature.

\section{Validation of Analytical Results}

We consider the stochastic image model in (27) with $M=64$ and $P=9$, where the target magnitudes $A_{p}$ are Rayleigh distributed with parameter $\sigma=1$ and the target locations are selected at random without replacement from the set of indices $m_{p} \in\{0,1, \ldots, M-1\}$. One column of this model is shown in Figure 2(a). Figures 2(b)-(f) show an experiment demonstrating the reinforcement of metric minima as an increased number of columns are included in the stochastic model. Plots of the objective function along each coordinate direction $f_{g}\left(\phi \boldsymbol{e}_{k}\right), k=0,1, \ldots, M-1$, (where, for comparison, the plots have been scaled to unity and the constant offset has been removed) are shown superimposed in Figure 2(b) for a single column of the stochastic model $(N=1)$. The metric minima are observed to be distributed with a large variance about $\phi=0$. Figure 2(c) shows plots of the objective function for $N=3$; here, the metric minima are distributed more closely about the origin. Further plots for $N=10, N=64$, and $N=256$ are shown in Figures 2(d), (e), and (f), respectively. These figures reveal that as more image columns are introduced, the contributions of the phase shifts $\angle Z_{k}[n]$ associated with each column average out, producing a stationary point at the origin.

To examine how the deviations of the metric minima from the origin decrease with an increasing number of columns, we examine the squared $\ell_{2}$-norm of the phase shifts as a function of $N:\left\|\boldsymbol{\Omega}^{[N]}\right\|_{2}^{2}$, where $\boldsymbol{\Omega}^{[N]}$ is a vector of the phase shifts from every coordinate direction $k=0,1, \ldots, M-1$. Figure 3 shows a plot (in log scale) of $\left\|\boldsymbol{\Omega}^{[N]}\right\|_{2}^{2}$ for the stochastic 


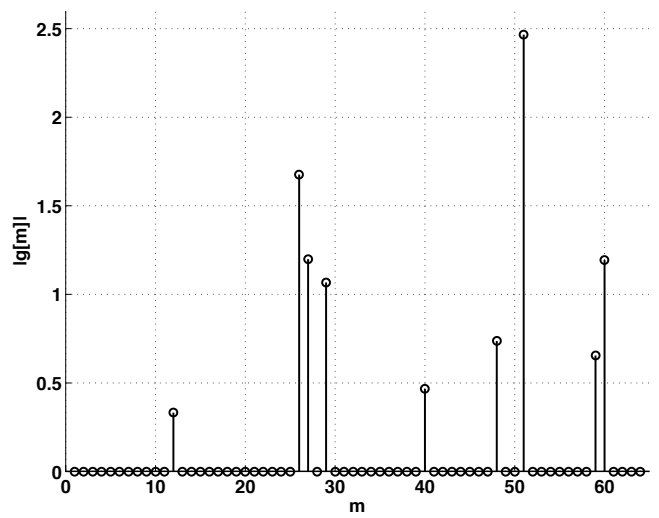

(a)

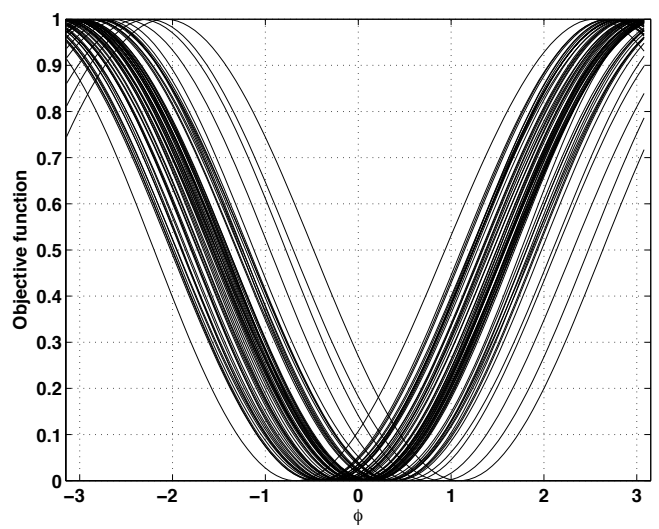

(c)

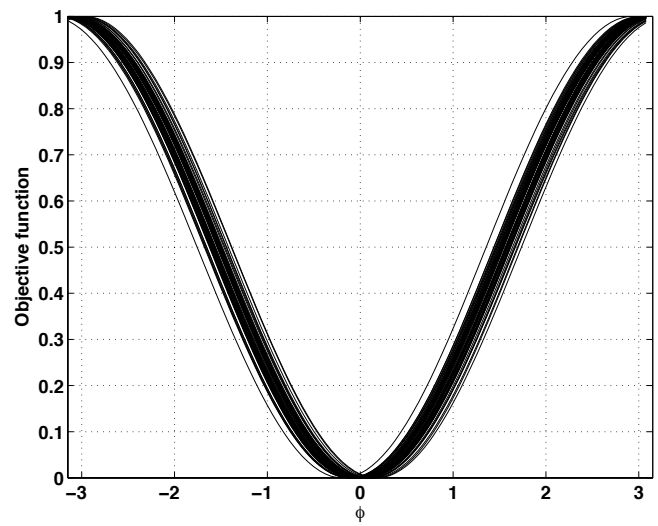

(e)

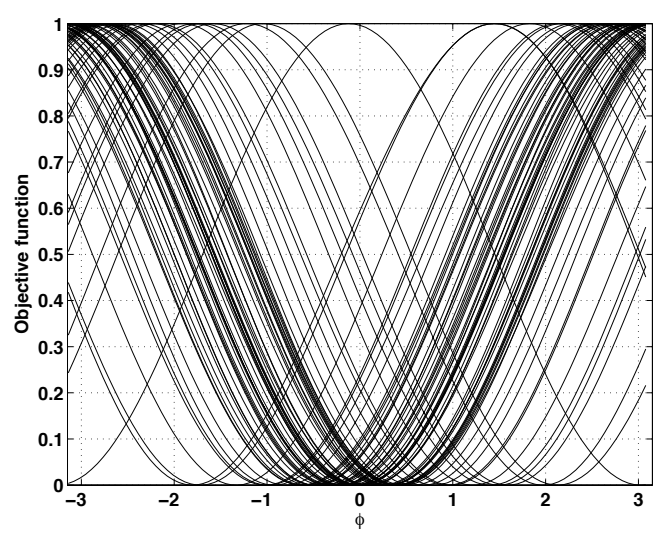

(b)

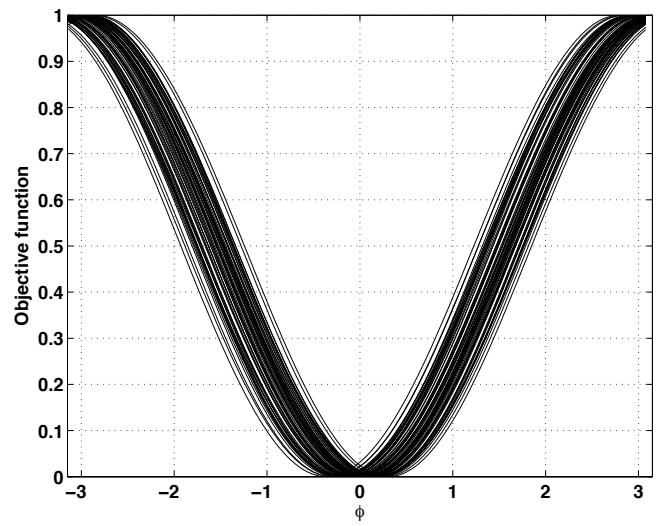

(d)

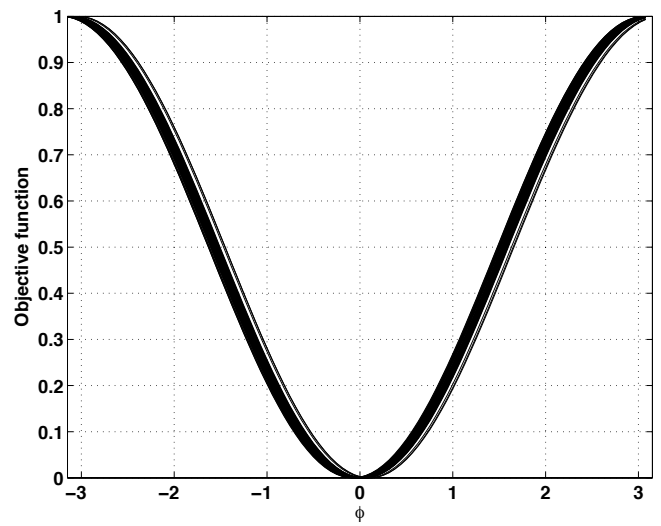

(f)

Fig. 2. Demonstration of the reinforcement of metric minima as an increased number of columns are included in the stochastic model. (a) One column of the stochastic model $(P=9, M=64)$. (b)-(f): Normalized plots of the objective function along each coordinate direction $\npreceq\left(\phi \boldsymbol{e}_{k}\right), k=0,1, \ldots, M-1$ (shown superimposed), where (b) $N=1$, (c) $N=3$, (d) $N=10$, (e) $N=64$, and (f) $N=256$.

model considered in the experiment of Figures 2(a)-(f); this plot is displayed using cross markers. It can be shown that $\left\|\boldsymbol{\Omega}^{[N]}\right\|_{2}^{2} \rightarrow M \sigma_{\Omega}^{2}[N]$ as $N$ increases. A plot of $M \sigma_{\Omega}^{2}[N] \sim \frac{1}{N}$ is shown in the dashed curve of Figure 3 for comparison. The plots reveal that the deviations from $\phi=0$ decrease sharply in the regime of small $N$. We can infer from this that the averaging effect takes place for a relatively small number of range bins containing dominant point targets; this provides justification for why metric-based methods work well for many practical SAR images.

In summary so far, we have demonstrated that, in general, a single column of the dominant point-targets model does not correspond to a stationary point. However, when the metric is evaluated on a large number of image columns, the metric minima along each $\boldsymbol{e}_{k}$ due to the phase shifts $\angle Z_{k}[n]$ reinforce each other to form a minimum at $\phi=0$. This averaging mechanism produces a stationary point at the origin, allowing the perfectly focused image to be properly restored. This observation is the key to understanding why metric-based approaches are successful. 


\section{Separable Approximation for the Multivariate OBJECTIVE FUNCTION}

\section{A. Derivation of the Local Separable Approximation}

We observe that locally about the origin, the objective function is approximately a separable function of the phase perturbations. That is, within a radius of the origin (e.g., $\|\phi\|_{2}<\xi, \xi \in \mathbb{R}$ ), the objective function is essentially a sum of terms that depend upon each phase component $\phi \boldsymbol{e}_{k}$ individually. To help formalize this finding, we derive an approximate expression for the multivariate objective function $f_{g}(\phi)$ that is separable in $\phi \boldsymbol{e}_{k}$, and we show that this approximation becomes more accurate as $\|\phi\|_{2}$ decreases. This approximation provides a local characterization of the second-order properties of the objective function.

Similar to our derivation of (18), we approximate the perturbed intensity as a function of the vector of phase perturbations $\phi$ using a first-order Taylor approximation. Analogous to (14), we write the phase-perturbed image as

$$
g_{\phi}[m, n]=g[m, n]+\varepsilon_{\phi}[m, n],
$$

where the image update can be expressed using (15) and (16) as

$$
\begin{aligned}
\varepsilon_{\boldsymbol{\phi}}[m, n] & =\sum_{k=0}^{M-1} \varepsilon_{\phi_{k} \boldsymbol{e}_{k}}[m, n] \\
& =\frac{1}{M} \sum_{k=0}^{M-1}\left(e^{j \phi_{k}}-1\right) G[k, n] e^{j 2 \pi k m / M},
\end{aligned}
$$

$\phi_{k}$ is the $k$-th component of $\phi$, and $\varepsilon_{\phi_{k} \boldsymbol{e}_{k}}[m, n]$ is defined in (15) (for fixed $n$ ). Using (42), and taking steps similar to those leading to (18) yields the approximation

$I_{\boldsymbol{\phi}}^{2}[m, n] \approx I^{2}[m, n]+4 \sum_{k=0}^{M-1} \Re\left\{I[m, n] g^{*}[m, n] \varepsilon_{\phi_{k} \boldsymbol{e}_{k}}[m, n]\right\}$.

The approximation in (44) is accurate when $\left|\varepsilon_{\phi}[m, n]\right|$ is small compared to $|g[m, n]|$. Analogous to (19), $\left|\varepsilon_{\phi}[m, n]\right|$ can be bounded from above as follows. Let $g$ be the model described by (5). Employing steps similar to those used to derive (19), from (43) we have $\left|\varepsilon_{\phi}[m, n]\right| \leq \frac{1}{M} \sum_{k=1}^{M-1}\left|\phi_{k} \| G[k, n]\right| \leq$ $\frac{1}{M}\|\phi\|_{2}\left\|G^{[n]}\right\|_{2}$, where the last inequality is a result of Cauchy-Schwarz and the superscript $[n]$ denotes the $n$-th column. Using $\left\|\boldsymbol{G}^{[n]}\right\|_{2}=\sqrt{M}\left\|\boldsymbol{g}^{[n]}\right\|_{2} \leq \sqrt{M P}\|\boldsymbol{g}\|_{\infty}$ we obtain

$$
\left|\varepsilon_{\boldsymbol{\phi}}[m, n]\right| \leq \sqrt{\frac{P}{M}}\|\boldsymbol{\phi}\|_{2}\|\boldsymbol{g}\|_{\infty},
$$

for all $m$ and $n$. Thus if

$$
\sqrt{\frac{P}{M}}\|\phi\|_{2} \ll 1
$$

then the approximation (44) is accurate. Note that this is true for sparse images $(P \ll M)$ and small phase perturbations.
Using the approximation (44), we have

$$
\begin{aligned}
f_{g}(\boldsymbol{\phi}) & =-\sum_{n=0}^{N-1} \sum_{m=0}^{M-1} I_{\phi}^{2}[m, n] \\
& \approx-\sum_{n=0}^{N-1} \sum_{m=0}^{M-1} I^{2}[m, n]-\sum_{k=0}^{M-1} \Re\left\{\left(e^{j \phi_{k}}-1\right) \zeta_{k}\right\} \\
& =\sum_{k=0}^{M-1} s_{g}\left(\phi_{k}\right)+\gamma .
\end{aligned}
$$

where

$$
\begin{gathered}
s_{g}\left(\phi_{k}\right) \stackrel{\text { def }}{=}-\left|\zeta_{k}\right| \cos \left(\phi_{k}+\angle \zeta_{k}\right), \\
\zeta_{k} \stackrel{\text { def }}{=} \frac{4}{M} \sum_{n=0}^{N-1} \sum_{m=0}^{M-1} I[m, n] g^{*}[m, n] G[k, n] e^{j 2 \pi k m / M},
\end{gathered}
$$

and $\gamma=-\sum_{n=0}^{N-1} \sum_{m=0}^{M-1} I^{2}[n, m]+\sum_{k=0}^{M-1} \Re\left\{\zeta_{k}\right\}$.

A consequence of (47) is that when the phase errors are small enough, we can optimize the multivariate objective function $f_{\tilde{g}}(\phi)$ by optimizing each phase component in $\phi$ independently. To demonstrate this, we first define $\xi$ to be the radius within which the separable approximation holds; i.e., (47) holds for all $\phi$ such that $\|\phi\|_{2} \leq \xi$. From (46), $\xi$ must satisfy

$$
\xi \ll \sqrt{\frac{M}{P}} .
$$

Using the relationship $f_{\tilde{g}}(\phi)=f_{g}\left(\phi+\phi_{e}\right)$, if the phase errors are small so that $\left\|\phi_{e}\right\|_{2}<\xi$, then from (47) we have

$$
\begin{aligned}
f_{\tilde{g}}(\phi) & \approx \sum_{k=0}^{M-1} s_{\boldsymbol{g}}\left(\phi_{k}+\phi_{e}[k]\right)+\gamma \\
& =-\sum_{k=0}^{M-1}\left|\zeta_{k}\right| \cos \left(\phi_{k}+\phi_{e}[k]+\angle \zeta_{k}\right)+\gamma
\end{aligned}
$$

for all $\phi$ satisfying $\left\|\phi+\phi_{e}\right\|_{2} \leq \xi$. An illustration of the region where the separable approximation holds is shown in Figure 4. Thus, from (50) we observe that the phase error estimate can be determined by performing a series of 1-D searches along each phase component, yielding the solution

$$
\hat{\phi}[k]=\phi_{e}[k]+\angle \zeta_{k},
$$

which is equal to the actual phase error function when the perfectly focused image corresponds to a stationary point $\left(\angle \zeta_{k}=0\right.$ for all $\left.k\right)$.

Using our analysis, we will derive an efficient procedure for performing the 1-D searches within the region of the separable approximation. We note that the resulting phase update has been discovered in [10] and [12] using different derivations and a different set of assumptions; in particular, the previous works do not exploit the separable nature of the objective function that we make use of here. Since the objective function is separble locally for all $\phi$ satisfying $\left\|\phi+\phi_{e}\right\|_{2} \leq \xi$, (50) can be expressed as

$$
f_{\tilde{g}}(\phi) \approx \sum_{k=0}^{M-1} s_{\tilde{g}}\left(\phi_{k}\right)+\gamma
$$




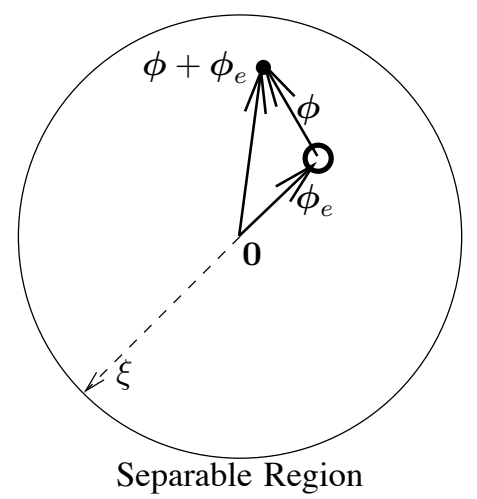

Fig. 4. Illustration of the region where the separable approximation holds The approximation holds for all $\phi$ such that $\left\|\phi+\phi_{e}\right\|_{2} \leq \xi$.

where

$$
s_{\tilde{\boldsymbol{g}}}\left(\phi_{k}\right) \approx-\left|\tilde{\zeta}_{k}\right| \cos \left(\phi_{k}+\angle \tilde{\zeta}_{k}\right)
$$

for some $\tilde{\zeta}_{k}$ (not yet determined). For (51) to match (50), we must demonstrate that

$$
\tilde{\zeta}_{k}=\zeta_{k} e^{j \phi_{e}[k]}
$$

Such a result implies $\left|\tilde{\zeta}_{k}\right|=\left|\zeta_{k}\right|$ and $\angle \tilde{\zeta}_{k}=\phi_{e}[k]+\angle \zeta_{k}$, thus reconciling the two expressions. Our goal is to show that

$$
\tilde{\zeta}_{k} \stackrel{\text { def }}{=} \frac{4}{M} \sum_{m, n} \tilde{I}[m, n] \tilde{g}^{*}[m, n] \tilde{G}[k, n] e^{j 2 \pi k m / M} .
$$

satisfies (53); in doing so, we establish a means for finding the minimizer along each $\phi_{k}$ without performing an explicit search. Noting that

$$
\tilde{g}[m, n]=g[m, n]+\varepsilon_{\boldsymbol{\phi}}[m, n]
$$

and

$$
\tilde{I}[m, n]=I[m, n]+2 \Re\left\{g^{*}[m, n] \varepsilon_{\boldsymbol{\phi}}[m, n]\right\}+\left|\varepsilon_{\boldsymbol{\phi}}[m, n]\right|^{2},
$$

we expand (54) as

$$
\begin{aligned}
\tilde{\zeta}_{k}= & \frac{4}{M} \sum_{n=0}^{N-1} G[k, n] e^{j \phi_{e}[k]} \\
& \sum_{m=0}^{M-1}\left(I[m, n]+2 \Re\left\{g^{*}[m, n] \varepsilon_{\boldsymbol{\phi}}[m, n]\right\}+\left|\varepsilon_{\boldsymbol{\phi}}[m, n]\right|^{2}\right) \\
& \left(g[m, n]+\varepsilon_{\boldsymbol{\phi}}[m, n]\right)^{*} e^{j 2 \pi k m / M} .
\end{aligned}
$$

Using $|g[m, n]| \gg\left|\varepsilon_{\boldsymbol{\phi}}[m, n]\right|$, which holds for the sparse model or when $\|\phi\|_{2}$ is small, the following approximation can be made:

$$
\begin{aligned}
\tilde{\zeta}_{k} & \approx \frac{4}{M} \sum_{n} G[k, n] e^{j \phi_{e}[k]} \sum_{m} g^{*}[m, n] I[m, n] e^{j 2 \pi k m / M} \\
& =\zeta_{k} e^{j \phi_{e}[k]} .
\end{aligned}
$$

Thus, when $\boldsymbol{g}$ corresponds to a stationary point, $\angle \zeta_{k}=0$ and $\angle \tilde{\zeta}_{k}=\phi_{e}[k]$ gives the unknown phase error. Note that (54) can be computed for all $k$ in $O(N M \ln M)$ operations using the DFT; this phase estimate update is similar to the one used in [10] and [12], but without higher-order terms that can be neglected for the sparse model or for small phase perturbations $\phi$.

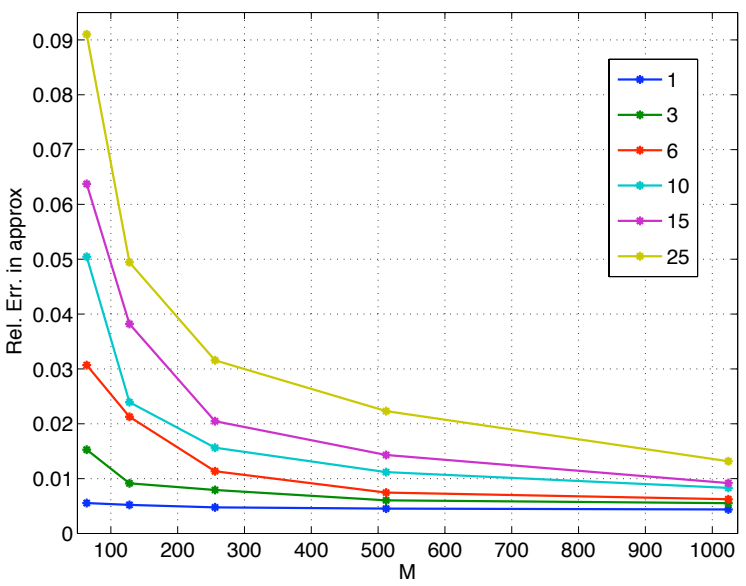

Fig. 5. Relative error in the intensity-squared approximation (44), where a small phase perturbation $\phi$ is applied to the sparse image model. Each plot shows the error as a function of $M$ for a fixed value of $P$ (the value of $P$ is indicated in the legend of each plot). As the bound in (46) predicts, the error decreases with decreasing $P$ and increasing $M$.

\section{B. Validation of the Separable Analysis}

To validate the bounds on $\phi$ in (46), we performed a Monte Carlo simulation where the relative error in the intensitysquared approximation in (44) was evaluated for sparse image models with different values of $P$ and $M$. The relative error is measured as $\left\|\boldsymbol{I}_{\phi}^{2}-\hat{\boldsymbol{I}}_{\phi}^{2}\right\|_{2} /\left\|\boldsymbol{I}_{\phi}^{2}\right\|_{2}$, where $\boldsymbol{I}_{\boldsymbol{\phi}}^{2}$ and $\hat{\boldsymbol{I}}_{\boldsymbol{\phi}}^{2}$ denote the actual and approximate squared intensity, respectively, subject to the phase perturbation $\phi$. For each value of $P$ and $M$, we performed ten trials where in each trial a different randomly generated phase perturbation with fixed statistics (the phase components are uniformly distributed between $-\pi / 8$ and $\pi / 8$ ) was applied. Figure 5 shows the results of the simulation when the phase perturbations are applied to the perfectly focused sparse image models. Each plot shows the average relative error as a function of $M$ for a fixed value of $P$ (the values $P=1,3,6,10,15,25$ and $M=64,128,256,512,1024$ were computed). As the bound in (46) predicts, the error decreases with decreasing $P$ and increasing $M$ (increasing sparsity).

In summary, the novel contribution of this section is that we have revealed the separable property of the intensity-squared objective function. This property provides justification for the success of the efficient optimization approaches in [10] and [12]; there has previously been little justification that applying the efficient phase update iteratively should result in rapid convergence to a local optimum.

\section{EXPERIMENTAL RESULTS}

To demonstrate how our analysis extends to actual SAR imagery, we performed numerical simulations using actual SAR images. We first consider the perfectly focused 600 by 600 pixel SAR image in Figure 6(a), which is formed from a given defocused image by applying an $I^{2}$-minimization autofocus routine [10]. Figure 6(b) shows normalized plots of the objective function using the image in (a) for each coordinate direction. As predicted by theory, the plots of the objective function for the perfectly focused image are 


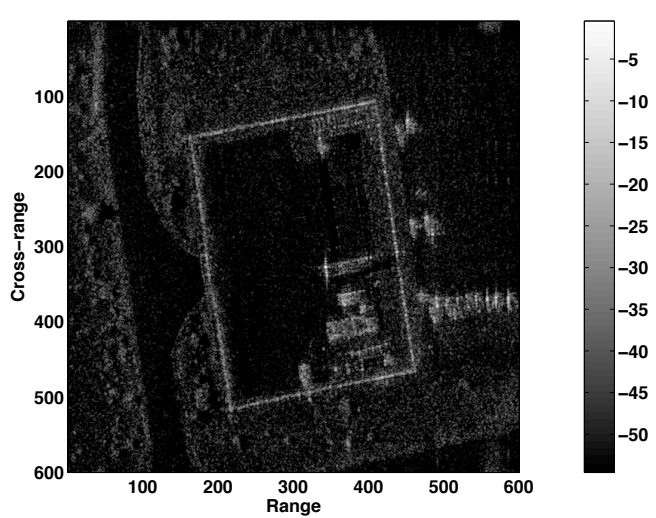

(a)

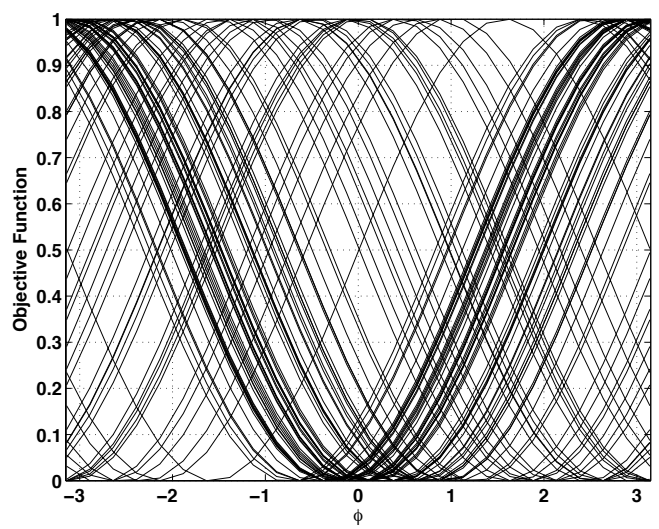

(c)

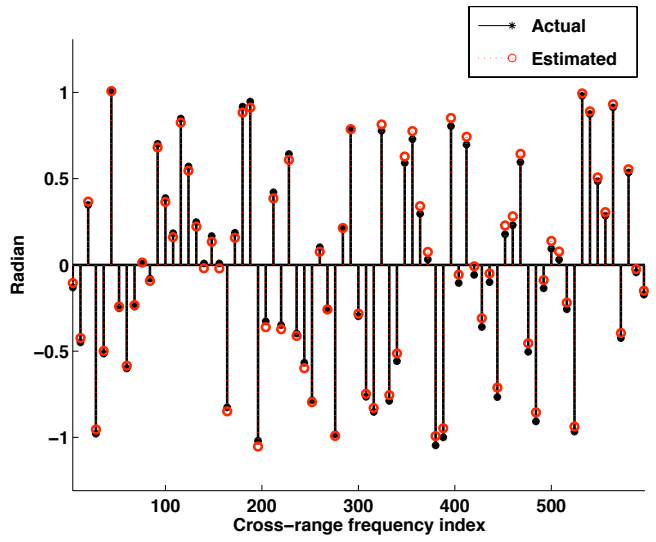

(e)

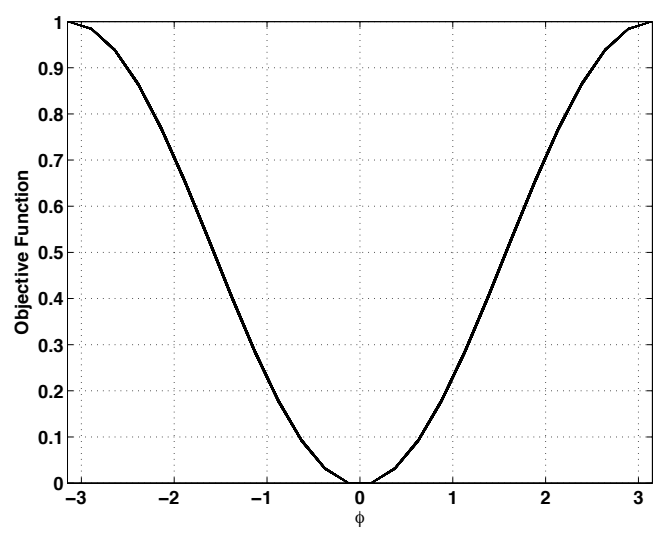

(b)

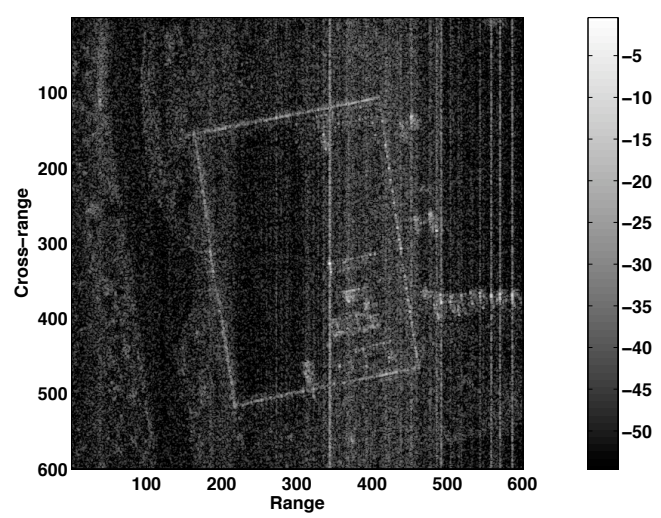

(d)

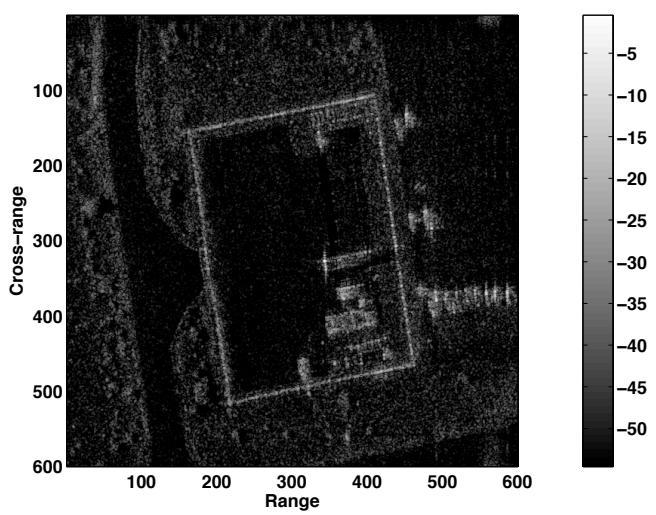

(f)

Fig. 6. Experiment with an actual SAR image. (a) Intensity of 600 by 600 pixel perfectly focused SAR image (displayed in dB), (b) normalized plots of the objective function along each coordinate direction $(k=0,1, \ldots, 600)$ for the image in (a), (c) normalized plots of the objective function for column 491 (for clarity, a subset of coordinate directions $k=3,11,19, \ldots, 595$ is shown), (d) defocused image where a small phase error has been applied (independent phase components uniformly distributed between $-\frac{\pi}{3}$ and $\frac{\pi}{3}$ ) to the image in (a), (e) applied phase error $\phi_{e}[k]$ (star markers) and phase estimate formed by performing simultaneous 1-D searches along each coordinate direction (circle markers) for $k=3,11,19, \ldots, 595$, and (f) image restored by applying the phase estimate in (e).

described by cosine functions, with minima at $\phi=0$ (note that since the all of the plots lie on top of each other, they resemble a single plot). To show that a single column of the image does not correspond to a stationary point, we evaluate plots of the objective function for column 491 (selected as a representative example), which are displayed in Figure 6(c). The objective function minima for the single column exhibit large variation about the origin. Figure 6(b) reveals that the effects of these minima are averaged out when the contributions of all the columns are taken into account, as demonstrated in our analysis.

We have demonstrated that locally about the origin, the objective function can be approximated as a separable function of the phase perturbations. To show this using actual data, we apply a small random phase error, with independent components uniformly distributed between $-\frac{\pi}{3}$ and $\frac{\pi}{3}$, to the 
perfectly focused image in Figure 6(a). The resulting defocused image is displayed in Figure 6(d). Figure 6(e) shows a plot of the phase shifts $\Omega_{k}$ for the defocused image (displayed in circle markers), determined by numerically performing 1 D searches along each phase component, superimposed on a plot of the applied phase errors $\phi_{e}[k]$ (displayed in star makers) for $k=3,11,19, \ldots, 595$. We see that these plots are in agreement, demonstrating that the effect of applying small phase perturbations to the perfectly focused image is to shift the objective function minima by an amount equal to the phase errors. Thus, in the local regime, the phase error can be determined by performing $M$ simultaneous one-dimensional searches along each coordinate direction (i.e., simultaneous coordinate descent). Figure 6(f) shows the restored image formed in this manner.

The image in Figure 6(a) consists of point-like features (e.g., corner reflections) against a low-return background, and thus the model in (5) is a good approximation to this actual image. To determine how the analysis extends to actual SAR images not well-described by the dominant point-targets model, we consider the perfectly focused terrain image of size 2027 by 2335 in Figure 7(a). As in the previous experiment, the perfectly focused image is formed from a defocused image by applying $I^{2}$-minimization autofocus. Figure 7(b) shows normalized plots of the objective function using the image in (a) for the subset of coordinate directions $k=$ $95,191,287, \ldots, 2015$. As in the experiment of Figure 6, the metric minima for each coordinate direction are located at $\phi=0$. Figure 7(c) shows plots of the objective function for a single column (column 680) for the same subset of coordinate directions. As expected, the figure reveals that the stationary point condition is not satisfied for the single image column.

From the analysis in (45), the separable approximation (47) is expected to hold well when the phase perturbations are small and the SAR image is sparse with a small number of point targets per range bin. To determine how well the separable approximation holds for the image in Figure 7(a), we apply a small random phase error with independent components uniformly distributed between $-\frac{\pi}{3}$ and $\frac{\pi}{3}$. The resulting defocused image is shown in Figure 7(d). As in the experiment using the previous image, we applied a single iteration of simultanous coordinate descent (i.e., $M$ independent 1-D searches) to the defocused image to determine an estimate of the phase error. The phase estimate is displayed in diamond markers in Figure 7(e), plotted with the actual phase error in star markers for $k=31,63, \ldots, 2015$. We observe that the phase estimate is not as accurate as the estimate produced in the experiment in Figure 6(e). This is expected, since the image in Figure 7(a) is not sparse like the previous image, and the separable approximation should hold less well. The plot denoted by circle markers in Figure 7(e) shows the result of applying three iterations of simultaneous coordinate descent; here, the phase error is recovered with a high degree of accuracy. We note that although the sparsity assumption holds weakly for the image in Figure 7(a), a rough estimate of the phase error is produced after a single iteration. However, when the applied phase errors are outside of the range $\left[-\frac{\pi}{3}, \frac{\pi}{3}\right)$, we have observed through experiments that the phase estimate obtained through a single iteration is not correct. This suggests that within a neighborhood of the local optimum, simultaneous coordinate descent can converge very quickly to the true phase estimate using a small number of iterations, even when the actual SAR image is poorly approximated by the ideal model (5). The simultaneous coordinate descent can be performed efficiently in an iterative fashion using the framework in [10].

\section{CONCLUSION}

We have presented analytical results providing new insight into metric-based methods for the restoration of SAR images. Considering the intensity-squared metric, and a dominant point-targets image model, we have derived a simple approximation for the resulting objective function. We demonstrated, in general, a single-column image model does not correspond to a stationary point. Thus, we saw that it is not the sharpness metric alone that makes these approaches successful, but the reinforcement of metric minima that takes effect with many image columns; it is here that the multichannel defocusing model of SAR autofocus is exploited implicitly. The necessity of the implicit multichannel assumption suggests that a means for exploiting the multichannel condition explicitly is needed. We are exploring this (i.e., see [22], [23]), where a linear algebraic formulation to the SAR autofocus problem, similar to those used in the past to study multichannel deconvolution problems, has been found to have great potential.

We have demonstrated that the multivariate objective function has a special structure by which it can be well approximated locally as a separable function of the phase perturbations. This structure is particularly evident near the perfectly focused image. As a consequence, when the phase errors are small, the metric minima are displaced from the origin by an amount equal to the phase errors. This allows the phase error to be estimated efficiently by performing $M$ 1-D searches along each coordinate direction simultaneously. Our analysis provides a formal justification for observations and assumptions in recent work [10], [12].

\section{ACKNOWLEDGMENT}

The authors would like to thank Dr. Charles Jakowatz and Sandia National Laboratories for the actual SAR data used in this paper, and Dr. Kyle Gallivan of Florida State University for stimulating discussions.

\section{REFERENCES}

[1] C. V. Jakowatz, Jr., D. E. Wahl, P. H. Eichel, D. C. Ghiglia, and P. A. Thompson, Spotlight-Mode Synthetic Aperture Radar: A Signal Processing Approach., Kluwer Academic Publishers, Boston, 1996.

[2] W. D. Brown and D. C. Ghiglia, "Some methods for reducing propagation-induced phase errors in coherent imaging systems - I: Formalism," J. Opt. Soc. Amer. A, vol. 5, pp. 924-942, 1988.

[3] D. C. Ghiglia and W. D. Brown, "Some methods for reducing propagation-induced phase errors in coherent imaging systems - II: Numerical results," J. Opt. Soc. Amer. A, vol. 5, pp. 943-957, 1988.

[4] C. E. Mancill and J. M. Swiger, "A map drift autofocus technique for correcting higher-order SAR phase errors," in Twenty-Seventh Annual Tri-Service Radar Symposium, Monterey, CA, June 1981, pp. 391-400.

[5] C. V. Jakowatz, Jr. and D. E. Wahl, "Eigenvector method for maximumlikelihood estimation of phase errors in synthetic-aperture-radar imagery," J. Opt. Soc. Am. A, vol. 10, no. 12, pp. 2539-2546, December 1993. 


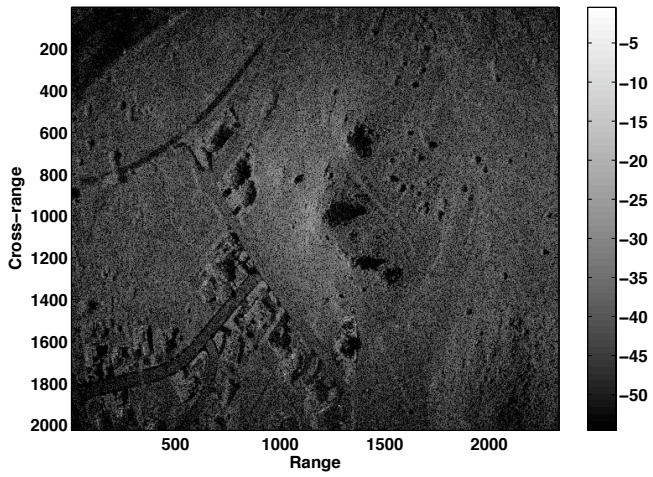

(a)

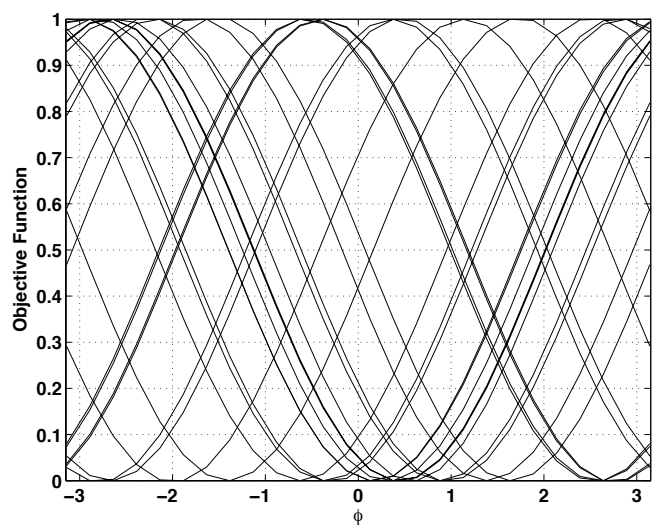

(c)

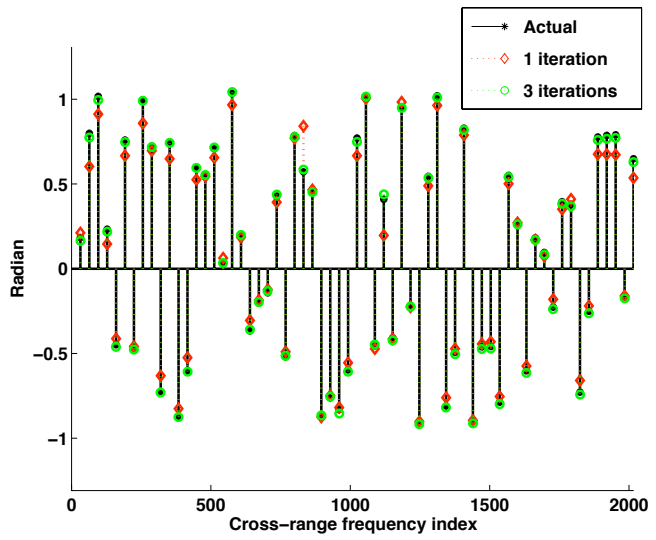

(e)

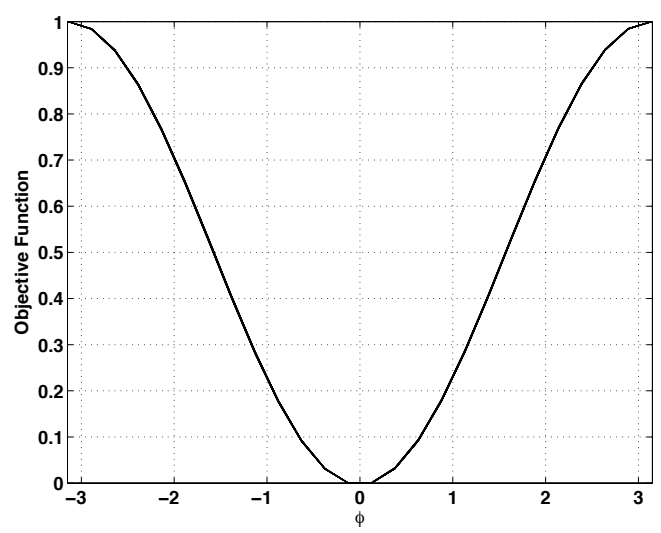

(b)

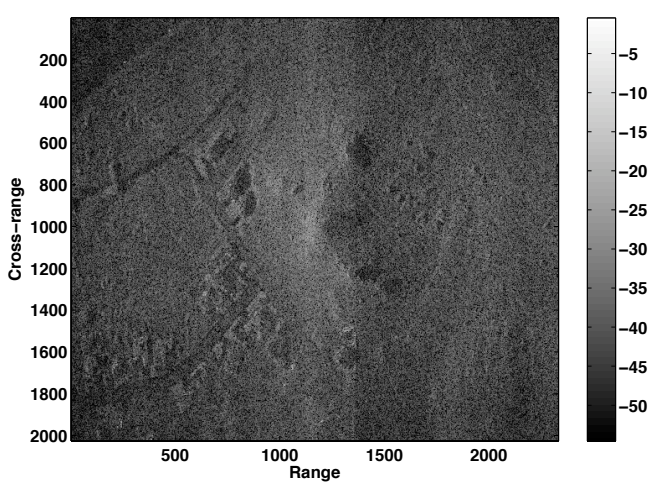

(d)

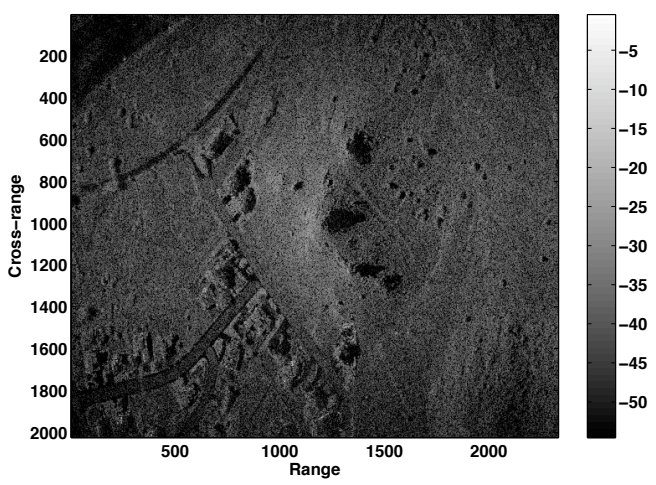

(f)

Fig. 7. Experiment with an actual SAR image. (a) Intensity of 2027 by 2335 pixel perfectly focused SAR image (displayed in dB), (b) normalized plots of the objective function along a subset of coordinate directions $(k=95,191,287, \ldots, 2015)$ for the image in (a), (c) normalized plots of the objective function for column 680 (where the subset of coordinate directions $k=95,191,287, \ldots, 2015$ is shown), (d) defocused image where a small phase error has been applied (independent phase components uniformly distributed between $-\frac{\pi}{3}$ and $\frac{\pi}{3}$ ) to the image in (a), (e) applied phase error $\phi_{e}[k]$ (star markers), and phase estimate obtained after one iteration of simultaneous coordinate descent (diamond markers) and three iterations (circle markers) for $k=31,63, \ldots, 2015$, and (f) image restored after one iteration of simultaneous coordinate descent.

[6] P. H. Eichel, D. C. Ghiglia, and C. V. Jakowatz, Jr., "Speckle processing method for synthetic-aperture-radar phase correction," Optics Letters, vol. 14, no. 1, pp. 1101-1103, January 1989.

[7] D. E. Wahl, P. H. Eichel, D. C. Ghiglia, and C. V. Jakowatz, Jr., "Phase gradient autofocus - a robust tool for high resolution SAR phase correction," IEEE Transactions on Aerospace and Electronic Systems, vol. 30, no. 3, pp. 827-835, July 1994.

[8] P. Tsakalides and C. L. Nikias, "High-resolution autofocus techniques for SAR imaging based on fractional lower-order statistics," IEE Proc.Radar, Sonar, Navig., vol. 148, no. 5, pp. 267-276, October 2001.

[9] J.R. Fienup and J. J. Miller, "Aberration correction by maximizing generalized sharpness metrics," J. Opt. Soc. Amer. A, vol. 20, no. 4, pp. 609-620, April 2003.

[10] T. J. Kragh, "Monotonic iterative algorithm for minimum-entropy autofocus," in Proc. Adaptive Sensor Array Processing (ASAP) Workshop, Lexington, MA, June 2006.

[11] L. Xi, L. Guosui, and J. Ni, "Autofocusing of ISAR images based on entropy minimization," IEEE Transactions on Aerospace and Electronic Systems, vol. 35, no. 4, pp. 1240-1252, October 1999.

[12] M. P. Hayes and S. A. Fortune, "Recursive phase estimation for image sharpening," in Image and Vision Computing New Zealand, Dunedin, New Zealand, 2005. 
[13] R. G. Paxman and J. C. Marron, "Aberration correction of speckled imagery with an image sharpness criterion," in Statistical Optics, Proceedings of the SPIE, San Diego, CA, 1988, vol. 976, pp. 37-47.

[14] F. Berizzi and G. Corsini, "Autofocusing of inverse synthetic aprture radar images using contrast optimization," IEEE Transactions on Aerospace and Electronic Systems, vol. 32, no. 3, pp. 1185-1191, July 1996.

[15] R. L. Morrison, Jr. and D. C. Munson, Jr., "An experimental study of a new entropy-based SAR autofocus technique," in Proc. of the IEEE International Conference on Image Processing, Rochester, NY, 2002, vol. II, pp. 441-444.

[16] H. Erdogan and J. A. Fessler, "Monotonic algorithms for transmission tomography," IEEE Transactions on Medical Imaging, vol. 18, no. 9, pp. 801-814, September 1999.

[17] R. L. Morrison, Jr., "Entropy-based autofocus for synthetic aperture radar," M.S. thesis, University of Illinois at Urbana-Champaign, Urbana, IL, 2002.

[18] R. A. Muller and A. Buffington, "Real-time correction of atmospherically degraded telescope images through image sharpening," Journal of Optical Society of America, vol. 64, no. 9, pp. 1200-1209, 1974.

[19] C. T. Kelley, Iterative Methods for Optimization, SIAM, Philadelphia, 1999.

[20] T. G. Kolda, R. M. Lewis, and V Torczon, "Optimization by direct search: New perspectives on some classical and modern methods," SIAM Review, vol. 45, no. 3, pp. 385-482, 2003.

[21] D. G. Luenberger, Linear and Nonlinear Programming., Kluwer Academic Publishers, Boston, 2003.

[22] R. L. Morrison, Jr. and M. N. Do, "Multichannel autofocus algorithm for synthetic aperture radar," in Proc. of the IEEE International Conference on Image Processing, Atlanta, GA, 2006, pp. 2341-2344.

[23] R. L. Morrison, Jr. and M. N. Do, "A multichannel approach to metricbased SAR autofocus," in Proc. of the IEEE International Conference on Image Processing, Genoa, Italy, 2005, vol. 2, pp. 1070-1073.

[24] D. C. Munson, Jr., J. D. O’Brien, and W. K. Jenkins, "A tomographic formulation of spotlight-mode synthetic aperture radar," Proceedings of the IEEE, vol. 71, no. 8, pp. 917-925, August 1983.

[25] R. L. Morrison, Jr., D. C. Munson, Jr., and M. N. Do, "Avoiding local minima in entropy-based SAR autofocus," in Proc. of the IEEE Signal Processing Workshop on Statistical and Array Processing, St. Louis, MO, 2003, pp. 454-457.

[26] D. C. Munson, Jr. and J. L. C. Sanz, "Image reconstruction from frequency-offset Fourier data," Proceedings of the IEEE, vol. 72, no. 6, pp. 661-669, June 1984.

[27] J. W. Goodman, Statistical Optics, John Wiley and Sons, New York, 1985.

[28] H. Stark and J. W. Woods, Probability, Random Processes, and Estimation Theory for Engineers., Prentice Hall, Upper Saddle River, NJ, 1994.

[29] W. Feller, An Introduction to Probability Theory and Its Applications., John Wiley and Sons, New York, 1968.

[30] J.R. Fienup, "Detecting moving targets in SAR imagery by focusing," IEEE Transactions on Aerospace and Electronic Systems, vol. 37, no. 3 , pp. 794-809, July 2001

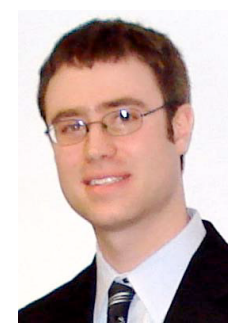

Robert L. Morrison, Jr. was born in Voorhees, New Jersey in 1977. He received the B.S.E. degree in electrical engineering from the University of Iowa in 2000, and the M.S. and Ph.D. degrees in electrical engineering from the University of Illinois at Urbana-Champaign in 2002 and 2007, respectively. Robert is currently a member of the technical staff at the Massachusetts Institute of Technology Lincoln Laboratory. His research interests include radar imaging, medical imaging, and signal processing.

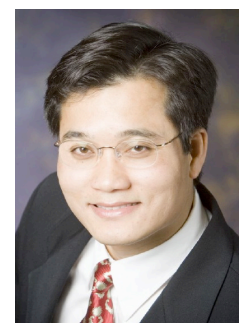

Minh N. Do was born in Thanh Hoa, Vietnam, in 1974. He received the B.Eng. degree in computer engineering from the University of Canberra, Australia, in 1997, and the Dr.Sci. degree in communication systems from the Swiss Federal Institute of Technology Lausanne (EPFL), Switzerland, in 2001.

Since 2002, he has been an Assistant Professor with the Department of Electrical and Computer Engineering and a Research Assistant Professor with the Coordinated Science Laboratory and the Beckman Institute, University of Illinois at Urbana-Champaign. His research interests include image and multi-dimensional signal processing, computational imaging, wavelets and multiscale geometric analysis, and visual information representation.

He received a Silver Medal from the 32nd International Mathematical Olympiad in 1991, a University Medal from the University of Canberra in 1997, the best doctoral thesis award from the Swiss Federal Institute of Technology Lausanne in 2001, and a CAREER award from the National Science Foundation in 2003. He was named a Beckman Fellow at the Center for Advanced Study, UIUC in 2006, and received of a Xerox Award for Faculty Research, College of Engineering, UIUC, in 2007. He is a member of the IEEE Signal Processing Society Signal Processing Theory and Methods and Image and MultiDimensional Signal Processing Technical Committees, and an Associate Editor of the IEEE Transactions on Image Processing.

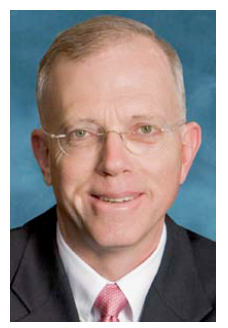

David C. Munson, Jr. David C. Munson, Jr. received the B.S. degree in electrical engineering (with distinction) from the University of Delaware in 1975, and the M.S., M.A., and Ph.D. degrees in electrical engineering from Princeton University in 1977, 1977, and 1979, respectively. From 1979 to 2003 he was with the University of Illinois at Urbana-Champaign, where he was the Robert C. MacClinchie Distinguished Professor of Electrical and Computer Engineering, Research Professor in the Coordinated Science Laboratory, and a faculty member in the Beckman Institute for Advanced Science and Technology. In 2003 he became Chair of the Department of Electrical Engineering and Computer Science at the University of Michigan, Ann Arbor. He currently is the Robert J. Vlasic Dean of Engineering at the University of Michigan.

Professor Munson's teaching and research interests are in the general area of signal and image processing. His research is focused on radar imaging, passive millimeter-wave imaging, and computer tomography. He has held summer industrial positions in digital communications and speech processing, and he has served as a consultant in synthetic aperture radar. He is co-founder of InstaRecon, Inc., a start-up to commercialize fast algorithms for image formation in computer tomography. He is affiliated with the Infinity Project, where he is coauthor of a textbook on the digital world, which is used in high schools nationwide to introduce students to engineering.

Professor Munson is a Fellow of the Institute of Electrical and Electronics Engineers (IEEE), a past president of the IEEE Signal Processing Society, founding editor-in-chief of the IEEE Transactions on Image Processing, and co-founder of the IEEE International Conference on Image Processing. In addition to multiple teaching awards and other honors, he was presented the Society Award of the IEEE Signal Processing Society, he served as a Distinguished Lecturer of the IEEE Signal Processing Society, he received an IEEE Third Millennium Medal, and he was the Texas Instruments Distinguished Visiting Professor at Rice University. 